\title{
Malte Daniljuk
}

\section{America's T-Strategy \\ Die US-Hegemonie und die Korrektur der \\ US-Außen- und Energiepolitik}

Die in den vergangenen Jahren eskalierten Konflikte in unmittelbarer Nähe der Europäischen Union (EU) (Ukraine) sowie die Veränderungen in der transatlantischen Außenpolitik können unter der Perspektive einer Neuordnung der weltweiten Energiepolitik besser verstanden werden. Infolge der Finanzkrise und mit dem Antritt eines demokratischen Präsidenten erlebten die USA eine deutliche Korrektur ihrer Außen- und Energiepolitik. Während wirtschaftsund handelspolitische Initiativen aus der Bush-Ära teilweise weitergeführt und fokussiert wurden, verschob sich die Sicherheitspolitik weg von einer direkten Präsenz im Rahmen eines „neuen Imperialismus“ (Harvey). Dies betrifft vor allem den Nahen und Mittleren Osten, wo sich ausgehend vom Irak-Krieg im März 2003 eine zeitweise Verwilderung abzeichnet. Die Region galt insbesondere wegen ihres Reichtums an fossilen Brennträgern als strategisch „entscheidend für US-Interessen“" (Cheney 2001: 131). Nach der Finanzkrise 2008 verfolgte die neue Regierung - zunächst auf dem nordamerikanischen Binnenmarkt und ab 2012 auch global - eine Strategie der sinkenden Energiekosten (O'Sullivan 2011). Neben einer zunehmenden Energieeffizienz wurden die dafür notwendigen Überschüsse mithilfe von neuen Fördertechnologien, insbesondere Horizontal Drilling und Hydraulic Fracturing (Fracking) geschaffen. Damit einher ging ein partieller militärischer Rückzug aus Konfliktregionen und ein insgesamt nachlassendes direktes Engagement im Greater Middle East, was Unilateralisten pauschal als „America's Retreat“ kritisieren (Stephens 2014).

Tatsächlich besteht der veränderte Kern außenpolitischen Handelns seitens der USA keineswegs in einem Rückzug, einem Versagen oder würde Grundlagen für American Decline-Debatten bieten. Vielmehr erleben wir eine insgesamt effizientere Außen- und Sicherheitspolitik, die darauf ausgerichtet ist, volkswirtschaftliche Kosten durch geringere physische Präsenz zu senken und weiche Faktoren im Kampf um weltweite Hegemonie zu stärken. Flankiert wird die minimalistische Militärstrategie durch diversifizierte Methoden der Außenpolitik, wirtschaftsund finanzpolitische Maßnahmen, eine zunehmende Einbindung multilateraler 
Organisationen und eine stärkere Verantwortung von regionalen Verbündeten wie der EU und den Golfstaaten (vgl. Obama 2014). Die Fähigkeit, im Rahmen der aktuellen Grand Strategy globale Entwicklungen aktiv zu steuern, lässt sich exemplarisch am Preissturz für Erdöl und den energiepolitischen Aspekten der kriegerischen Konflikte in den letzten Jahren illustrieren.

\section{Die Grundlage des Fracking-Booms}

Der Fracking-Boom ist weder ein spontanes konjunkturelles Ereignis noch eine technische Revolution, sondern das Ergebnis energiepolitischer Steuerungsmaßnahmen, die spätestens im Jahr 2005 unter der Regierung George W. Bush konkrete Formen annahmen. Die US-Bundesregierung hält einen hohen Anteil an Schiefervorkommen in der staatlichen Ölreserve vor und ließ seit Jahrzehnten die Forschung und Entwicklung von Schieferressourcen vorantreiben. Explizites Ziel dieser Strategie war es, den globalen Rohölpreis zu senken und eine größere außenpolitische Handlungsfreiheit zu erreichen. Mit dem Energy Policy Act von 2005 ermöglichte die damalige US-Regierung einen vereinfachten Zugang zu Förderlizenzen auf Land der Bundesbehörden, sie senkte radikal die Umweltstandards und erleichterte die Kapitalisierung von Erschließungsprojekten. Die Entwicklung fand ihren Ausgangspunkt 2004, mitten in der Peak-Oil-Debatte. Zu dieser Zeit sank infolge des Irak-Kriegs nicht nur die dortige Ölförderung. Alle Prognosen gingen davon aus, dass die konventionelle Förderung in den USA dramatisch zurückgehen würde. Gegenüber dem Vorjahr erhöhten sich die internationalen Preise für Rohöl erstmalig um zehn auf etwa 43 US-Dollar - eine Steigerung, die in den kommenden zehn Jahren anhalten sollte.

Im März 2004 veröffentlichte der Deputy Assistant Secretary for Petroleum Reserves eine erste umfangreiche Studie zu America's Oil Shale Resource (Johnson 2004). Mit Blick auf den Nachbarn Kanada, wo die Ausbeutung von Teersanden bereits ein Niveau von etwa einer Million Barrel Rohöl pro Tag erreicht hatte, empfahlen die Autoren, die kommerzielle Nutzung der US-Schieferölvorkommen vorzubereiten. Zu diesem Zeitpunkt wurden die bekannten Reserven mit über zwei Billionen Barrel angegeben, die zum Großteil seit 1912 von der Naval Petroleum and Oil Shale Reserves (NPOSR) verwaltet wurden. „Mit einer zwischen Industrie und Regierung koordinierten Kampagne ist es möglich, dass bis zum Jahr 2011 eine Ölschieferindustrie entsteht, die als aggressives Ziel eine Tagesproduktion von 2 Millionen Barrel hat und letztlich, in 2020, fähig sein könnte, 10 Millionen Barrel am Tag zu fördern." (Ebd.: 10; diese und alle weiteren ZitatÜbersetzungen durch den Verfasser) 
Das Ziel einer solchen Initiative sahen die Autoren nicht vordergründig darin, den nordamerikanischen Energiebedarf zu decken. Stattdessen gingen sie davon aus, dass ein zusätzliches Rohölangebot im skizzierten Umfang den weltweiten Preis für Rohöl drücken konnte. Zudem würden reduzierte Importe und ein sinkender Preis für Rohöl einen starken positiven Effekt auf das US-amerikanische Bruttoinlandsprodukt sowie die Handelsbilanz haben. Weiterhin konnte eine Schieferölindustrie dazu beitragen, dass die Förderung von Erdgas wieder stiege. Die Autoren dachten von Anfang daran, mit dieser Initiative auch einen entsprechenden Technologievorsprung für die US-Wirtschaft zu erreichen: „Rund 26 andere Länder weisen kommerziell nutzbare Mengen von Ölschiefer auf. Die in den USA entwickelte Technologie könnte dort angewandt werden. Die neue Technologie kann die Grundlage für neue Geschäftsmöglichkeiten sein.“ (Ebd.)

Die inzwischen berüchtigten technischen Methoden Horizontal Drilling und Hydraulic Fracturing könnten der Studie zufolge zusammen mit neuen geologischen Analyseverfahren beim damals aktuellen Preisniveau eine rentable Förderung erreichen. Sie verortete das größte Hindernis für die zu entwickelnde neue Industrie im Bereich der Finanzierung. Um der Energieindustrie den Anfang zu erleichtern, empfahlen die Autoren Steuernachlässe, Hilfen bei der Landvergabe und beim Aufbau der nötigen Infrastrukturen sowie beim Zugang zu Wasser. Insgesamt prognostizieren sie eine mit der Offshore-Förderung vergleichbare Rentabilitätsgrenze ab 25 US-Dollar Investition pro Barrel. Dabei wiesen sie anhand der kanadischen Teersandförderung darauf hin, dass die technische Effizienz mit zunehmender Erfahrung und wirtschaftlichem Erfolg zunehmen würde.

Kurz darauf stellte das Department of Energy (DOE) eine konkrete Roadmap vor. Innerhalb von 12 Monaten sollte ein konkreter Einstieg in die FrackingIndustrie erreicht werden (Dammer 2004). Die Bundesregierung in Washington wollte so schnell wie möglich etwaige rechtliche und administrative Hürden für potenzielle Investoren abbauen. Alle Institutionen wurden angehalten, Genehmigungen und Umweltbewertungen zügig zu erstellen. Mit öffentlichen Mitteln sollte die Forschung und Entwicklung der neuen Fördertechniken unterstützt werden. Außerdem sollten wirtschaftliche und steuerliche Anreize gesetzt und die Unternehmen mit der notwendigen Infrastruktur unterstützt werden. Das DOE ging davon aus, dass fast 80 Prozent der gesamten Ölschiefer-Ressourcen von Bundes- und Landesbehörden verwaltet werden. Der Mineral Leasing Act von 1920 hatte die Anzahl und den Umfang der an private Firmen zu vergebenden Lizenzen begrenzt und sollte entsprechend überarbeitet werden. Außerdem wurden die Bundesstaaten aufgefordert, Lösungen für den extrem hohen Wasserbedarf der Fracking-Industrie zu finden.

Da die Ölschieferproduktion außerordentlich hohe Front-End-Investitionsund Betriebskosten aufweist, außerdem lange Vorlaufzeiten zwischen Investitionen 
und operativen Erträgen bestehen, sollten politische Maßnahmen entwickelt werden, um das „Anlagerisiko zu reduzieren“. Dafür müsse eine enge Kommunikation zwischen Politik, Industrie und Investment Community entwickelt werden. Um all das mit hunderten beteiligten Behörden und Verwaltungsgliederungen zu erreichen, setzte das DOE eine Federal Oil Shale Task Force ein, für die unverzüglich ein 12-monatiger Projektplan für das gesamte Jahr 2005 aufgestellt wurde.

Im August 2005 unterzeichnete Präsident George W. Bush den Energy Policy Act of 2005, der sich laut Titel mit „unkonventionellen“ Ölreserven wie Schieferöl und Teersänden befasst. Tatsächlich handelte es sich um ein Ermächtigungsgesetz, das der gesamten Energieindustrie, also auch den Betreibern von Atomkraftwerken und der Kohleindustrie, bestehende Auflagen aus dem Weg räumte. Die Öl- und Gasindustrie wurde von Wasserschutzgesetzen, SchadstoffRichtlinien für Ölquellen und Genehmigungsverfahren für Infrastrukturen auf öffentlichem Grund und Boden befreit. Außerdem sollten Energieunternehmen mit einem Gesetz für wirtschaftliche Krisenzeiten gegen wirtschaftliche Ausfälle abgesichert werden. Sämtliche Bereiche von Big Energy wurden zudem mit erheblichen Steuererleichterungen bedacht - insgesamt 15,5 Milliarden US-Dollar pro Jahr. Umweltauflagen wurden gesenkt und bisher umstrittene Bereiche erlaubt, etwa Bohrungen in tiefen Meeresgewässern. Selbst die Washington Post sprach von einer „breit angelegten Sammlung von Subventionen für die amerikanischen Energieunternehmen“. Laut ihren Berechnungen summierten sich darin gewährten Subventionen und Steuerentlastungen auf insgesamt 85 Milliarden US-Dollar (Grunwald/Eilperin 2005).

\section{Obamas Agenda Surfing}

Zwischen 2005 und 2011 weitete sich die Energieproduktion in den USA radikal aus. Alleine die Beschäftigungszahlen im Sektor Oil and Gas Operations verdoppelten sich beinahe. „Der Boom bei der Förderung von Erdöl und Erdgas aus Schieferformationen wurde nach 2008 zu einem bedeutenden Faktor." (Brown 2013) Seitdem die Shale-Politik im Jahr 2005 einsetzte, wuchsen die Beschäftigungszahlen in den vier Bundesstaaten mit den höchsten Anteilen von Öl und Gas. Am stärksten nahm die Beschäftigung in North Dakota und Texas zu, also dort, wo die Produktion von Shale und Tight Oil (Schiefergas und Gas in dichtem Gestein) die höchsten Steigerungen zu verzeichnen hatte. Während die Beschäftigungszahlen in den gesamten USA zwischen 2006 und 2012 um 0,05 Prozent pro Jahr zurückgingen, stiegen sie in diesen beiden Staaten um 3,4 und 1,5 Prozent (ebd.).

2008 stellten Barack Obama und Joe Biden im US-Wahlkampf ein neues energiepolitisches Konzept vor, das zunächst auf einen ökologischen Umbau der 
Wirtschaft abzuzielen schien (Obama/Biden 2008). Von den umweltpolitischen Aspekten dieser Pläne blieb nach ihrem Amtsantritt so gut wie nichts übrig, selbst wenn bestimmte Vorgaben wie niedrigere Energiepreise und größere Unabhängigkeit von Importen sehr wohl erreicht wurden - allerdings auf ganz anderem Weg als zunächst angekündigt. Das Biden-Obama-Papier hatte sich außerdem den heimischen Ölquellen gewidmet. Hier erwähnten die Autoren, dass etwa 85 Milliarden Barrel „technisch förderbares" Rohöl in bereits bekannten Feldern stecken. Spätestens mit ihrem Amtsantritt, als die Zahlen für die inländische Öl- und Gasförderung für das Jahr 2008 vorlagen, dürfte die neue Regierung verstanden haben, dass sie von einem energiepolitischen Projekt der Bush-Ära profitieren würde, wenn auch auf Kosten der eigenen ambitionierten Umweltund Klimaziele.

Als die Regierung im März 2011 ihre energiepolitische Strategie vorstellte, nahmen die heimischen Öl- und Gasreserven sowie ihre Förderung mithilfe neuer Technologien bereits den größten Raum ein (Obama 2011). Gegenüber dem Jahr 2008 hatte sich die heimische Ölförderung bereits um gut 1,5 Millionen Barrel am Tag erhöht - erstmals seit 1985 und gleich um eine durchaus beeindruckende Menge. Die Obama-Regierung profitierte nun von einem Projekt, das abseits der öffentlichen Debatte von George W. Bush angelegt worden war. Hatten die Demokraten noch im Wahlkampf stark daraufgesetzt, gemeinsam mit der jungen Silicon-Valley-Kapitalfraktion innovative Energielösungen vorzubereiten, vollzog sich nun eine radikale Umorientierung hin zur alten Öl- und Gasindustrie.

Entsprechend lautete der wichtigste Punkt jetzt: „Wir müssen amerikanische Vermögenswerte, Innovationen und Technologie bereitstellen, sodass wir hier zu Hause sicher und verantwortungsvoll mehr Energie fördern und Marktführer in der globalen Energiewirtschaft werden." (Obama 2011) Obama wies darauf hin, dass die Zuwächse in der heimischen Öl- und Gasförderung hauptsächlich aus den Shale-Vorkommen stammen. „Um eine stabile Förderung und Erschließung von Ressourcen des Landes zu fördern, hat die staatliche Verwaltung in den vergangenen zwei Jahren Millionen Hektar an öffentlichen Flächen und Gewässern aus dem Bundeseigentum im Rahmen von Öl- und Gas-Leasing-Verträgen vergeben." (Ebd.) Insgesamt seien in 2010 und 2011 fast 60 Lizenzen für die Öl- und Gasförderung alleine bei der On Shore-Förderung vergeben worden, was etwa einem Drittel der verfügbaren Flächen gleichkommt. Obama versprach schnellere Vergabeprozeduren bei der Verpachtung oder dem Verkaufvon Förderflächen und spezielle Regelungen für Abgaben und Steuern im Öl- und Gassektor: „Obwohl der Preis von Öl und Gas den wichtigsten finanziellen Anreiz für Lizenznehmer darstellt, um ihre Projekte voranzubringen, können unterschiedliche Gebühren und Lizenzstrukturen die Entwicklung stärker beschleunigen." (Ebd.) Die USStrategie sollte nun darin bestehen, die inländische Förderung zu erhöhen, die 
Energieeffizienz zu steigern und Förderländer weltweit dabei zu unterstützen, ihre Produktion auszuweiten. „Anschließend an die Entwicklung von neuen Techniken zur Gewinnung von Schiefergas in den USA gründet das Außenministerium die Global Shale Gas-Initiative, die das Potenzial verschiedener Länder hinsichtlich der Schiefergasproduktion bewertet und die dortigen Regierungen unterstützt, Regeln für den Handel, die Sicherheit und den Umweltschutz festzulegen, die eine vorteilhafte Entwicklung dieser Ressource ermöglichen." (Ebd.)

\section{Der Impact in Zahlen}

Der energiepolitische Effekt der eingeleiteten Maßnahmen hat die globale Energielandschaft gründlich verändert. Seit 2014 sind die USA nicht mehr nur größter Erdgas- sondern größter Erdölproduzent - erstmals seit 1979. Im letzten Jahr der Bush-Ära (2008) hatte die US-Produktion mit 6,8 Millionen Barrel pro Tag ihren vorläufigen historischen Tiefpunkt erreicht. ${ }^{1}$ Seitdem steigt der zusätzliche Ausstoß fast exponentiell. Die Importe in die USA gehen hingegen sehr schnell zurück, was mit dem deutlichen Anstieg der inländischen Energieförderung zusammenhängt. Von 2007 bis 2014 sparte das Land 4,41 Millionen Barrel pro Tag an Einfuhren. Dabei verschoben sich die Herkunftsländer deutlich: Während die Importe aus sämtlichen übrigen Regionen zurückgehen, profitiert vor allem Kanada von einem immer größeren Absatz in die USA (2014: 167,7 Millionen Tonnen; 2013: 154,5). Die nächstwichtige Herkunftsregion bleibt Lateinamerika, wobei der größte Einzelposten mit 41,8 Millionen Tonnen (2013: 45,6) aus Mexiko stammt. Aus dem Mittleren Osten importieren die USA noch 93 Millionen Tonnen (2013: 100,1), aus Westafrika 16,9 (2013: 31,5). Insgesamt lässt sich für die USA also eine ausgeprägte Regionalisierung des Energiebezugs feststellen.

Ein Vergleich zwischen konventioneller Förderung und Fracking-Produktion in den sechs Regionen, für die beide Datensätze vorliegen (Bakken, Eagle Ford, Haynesville, Marcellus, Niobrara und Utica), zeigt, dass die Entwicklung ganz klar von den neuen Fördertechnologien angetrieben ist. Während die absolute Menge des konventionell geförderten Erdöls über die 7,5 Jahre fast genau konstant blieb, gehen die beeindruckenden Steigerungsraten ganz klar auf das Hydraulic Fracturing zurück.

Bei aller Euphorie, die viele angesichts des „Fracking-Wunders“ äußerten, sollte jedoch nicht vergessen werden, dass dieses nur unter der Bedingung eines sehr hohen Rohölpreises zustande kam. Die Volkswirtschaft insgesamt leidet unter

1 Alle Zahlen, wenn nicht anders angegeben, aus BP Statistical Review 2015. URL: http:// www.bp.com, Zugriff: 23.10.2015. 
steigenden Rohölpreisen. Die zunehmende Förderung bei hohen Preisen war daher eine auf die Öl- und Gasförderer beschränkte Konjunktur, da sie an ihnen gut verdienten. Selbst den Raffineriebetrieben schadeten die hohen Energiepreise. ${ }^{2}$

In der geografischen Verteilung drückt sich das so aus, dass sich die Wirtschafts- und Sozialdaten in 43 Bundesstaaten - darunter auch in denen mit Raffinerien - verschlechtern, sobald der Ölpreis steigt. Dabei gilt die Daumenregel, dass ein Anstieg des Ölpreises um 10 Prozent die Beschäftigung um 0,2 Prozent senkt. Dagegen profitieren die Bundesstaaten mit einer Förderindustrie: Alaska, Louisiana, New Mexico, North Dakota, Oklahoma, Texas, West Virginia und Wyoming (Brown 2013).

Für den Zeitraum 2008 bis 2011, also zum Beginn des Shale-Booms, veröffentlichte die Energy Information Administration (EIA) die vergebenen Förderlizenzen für die 18 damals wichtigsten Regionen (EIA 2011). Die 188 Genehmigungen teilten sich auf immerhin 75 Firmen auf, von denen die meisten allerdings nur ein oder zwei Flächen ausbeuteten, d.h. die Fracking-Revolution wurde tatsächlich zumeist von regional aktiven mittelständischen Firmen getragen. Von den im FT500 vertretenen Multis war zu diesem Zeitpunkt namentlich nur die Occidental Petroleum Company mit einem größeren Feld vertreten.

Das auffälligste Merkmal an dieser Entwicklung ist, dass der folgende Preissturz für Erdöl auf dem Weltmarkt keinen unmittelbaren Effekt auf das USFörderniveau hatte. Ab Juni 2014 stiegen sowohl die Erdöl- wie auch die Erdgasförderung weitere zehn Monate unvermindert an. Dies ist um so erstaunlicher, als die Bohrlochaktivitäten unmittelbar mit den Weltmarktpreisen für Erdöl zurückgingen. Sie erreichten ihr Maximum mit 1.308 aktiven Bohrlöchern in den sieben wichtigsten Regionen im Oktober 2014, um dann radikal abzufallen. Im Juni 2015 - ein Jahr nach dem Beginn des Preissturzes - lagen sie in den untersuchten Regionen nur noch bei 578. Die Ursache für diese Diskrepanz zwischen Bohraktivitäten und Ausstoß liegt in der schnell zunehmenden Produktivität der aktiven Bohrlöcher. Wie bereits im Jahr 2005 vorausgesagt, verzeichnete die Ölund Gasförderung mithilfe der unkonventionellen Methoden Effizienzgewinne: Am Beginn der erfassten Daten im Januar 2007 wurden aus einem Bohrloch durchschnittlich 39 Barrel am Tag gefördert. Im August 2015 waren es 380 Barrel täglich, d.h. in acht Jahren hatte sich der Ausstoß pro Bohrloch fast um den Faktor 10 vervielfacht. 2014 wurden mit weniger als der Hälfte der Bohrlöcher knapp eine Million Barrel Rohöl am Tag zusätzlich gefördert. Die andere Hälfte gehört zu unrentablen Projekten bzw. handelt es sich um Erschließungsbohrungen, deren zukünftige Ausbeute zu diesem Zeitpunkt noch nicht gesichert war.

2 Bei „Upstream“ (Förderung) und „Downstream“(Verarbeitung, Verkauf) handelt es sich grundsätzlich um unterschiedliche Branchen und Firmen. 


\section{Außen- und sicherheitspolitische Konsequenzen}

Nachdem sich die Zuwächse in der heimischen Energieförderung klar abzeichneten, begannen die energiepolitischen Fachdebatten das politische Potenzial des „Fracking-Wunders“ abzuschätzen. Anhand des Elitendiskurses lässt sich rekonstruieren, dass sich die verschiedenen Politikebenen frühzeitig über die geostrategischen Potenziale einer expandierenden heimischen Energieförderung verständigten. Im Juni 2012 meldete sich Leonardo Maugeri, ehemaliger Manager des italienischen Energieunternehmens Eni, mit einer Analyse für das Belfer Center for Science and International Affairs der Harvard University zu Wort. Zu diesem Zeitpunkt erreichte die nur auf Fracking zurückzuführende Erdölförderung in den USA erstmals die Grenze von zwei Millionen Barrel. Auf Grundlage des relativ kontinuierlichen Wachstums der weltweiten Fördermenge an Öl und Gas zwischen den Jahren 2000 und 2011 prognostizierte Leonardo Maugeri einen erheblichen Überschuss an geförderten fossilen Energieträgern und somit einen deutlichen Preisverfall auf etwa 70 US-Dollar pro Barrel bis zum Jahr 2020. Zu diesem Zeitpunkt lag der internationale Durchschnittspreis deutlich über 100 US-Dollar. ${ }^{3}$ Seine Argumentation stützt sich im Wesentlichen auf die durch DeConventionalization zunehmende Förderung auf dem amerikanischen Kontinent, wo sich damals 70 Prozent der bekannten unkonventionellen Ressourcen befinden. Deshalb argumentierte Maugeri, dass eine deutliche Machtverschiebung hin zur „westlichen Hemisphäre“ einsetzen würde. Zwar würden die USA niemals vollständig unabhängig vom globalen Ölmarkt und dem Mittleren Osten sein, aber die Region würde „nur noch eine Säule des Geschehens“ sein. Hingegen würde sich abzeichnen, dass die Opec durch das globale Angebot und die zunehmende Förderung im Irak unter Druck geriete. Zudem werde sich Asien zum entscheidenden Markt für Energie entwickeln. China, so Maugeri, würde nicht nur im Mittleren Osten und in Afrika der politische Konkurrent der USA. Womöglich könne das Land seinen Einfluss sogar auf Länder wie Venezuela und Kanada ausdehnen.

Ebenfalls im Jahr 2012 verfasste der Special Envoy and Coordinator for International Energy Affairs des US-Außenministeriums, David Goldwyn, einen ausführlichen Hintergrundbeitrag in der New York Times (Goldwyn 2012). Er arbeitete bereits seit 1991 für das Außenministerium und war seit 1999 schwerpunktmäßig mit Energiefragen beschäftigt. Seit 2007 gehörte er zum Council on Foreign Relations, wo er in der Task Force zu National Security Consequences of U.S. Oil Dependency mitarbeitete. Die aktuelle Entwicklung werde es den USA erlauben, sich stärker nach innen zu orientieren und internationalen Angelegenhei-

3 Durchschnittliche Ölpreise 2011 in US-Dollar: Dubai 106,18; Brent 111,26; Nigerian 113,65; WTI 95,04. 
ten weniger Interesse entgegenzubringen - einschließlich dem Nahen und Mittleren Osten, argumentierte Goldwyn. Allerdings habe die neue Unabhängigkeit ihre Grenzen: „Die Probleme der Preisvolatilität, eine Vielfalt des Angebots und die Verpflichtung, unseren Freunden und Verbündeten zu helfen, sich frei von monopolistischer Preisgestaltung oder Zwangsregelungen zu versorgen, werden in den kommenden 20 Jahren genau so entscheidend bleiben, wie sie es heute sind." (Ebd.) Goldwyn illustrierte die teilweise widersprüchliche Situation anhand der gegen den Iran verhängten Sanktionen: So habe das seit 2011 reduzierte Ölangebot aus dem Iran dafür gesorgt, dass der Brent-Preis sich in Richtung 120 US-Dollar bewegte und unter anderem die Benzinpreise in den USA erhöhte.

Mit Blick auf verschiedene Förderregionen sah David Goldwyn die zukünftige Außenpolitik eher in der Verantwortung, eine ausreichende globale Versorgung sicherzustellen und größere Produktionsausfälle zu vermeiden. Die USA sollten anderen Ländern sogar helfen, ihre eigenen Rohstoffressourcen intensiver zu fördern, indem das eigene technische und politische Know-how zugänglich gemacht wird. „Eine höhere Produktion wird dazu beitragen, die Preise moderat zu halten und die Versorgung zu diversifizieren." (Goldwyn 2012) Natürlich seien Investoren nicht an moderaten Ölpreisen interessiert. Aber aus einer außenpolitischen Perspektive stelle zunehmender Wettbewerb die beste Versicherung gegen „die schlimmsten Tendenzen der Petro-Staaten“ dar. Erstmals sprach er die Perspektive an, dass die USA zukünftig Energie exportieren könnten - etwa nach Europa. Die Autonomie, beispielsweise der osteuropäischen Länder, und die Freiheit von Europa, sich seine Energielieferanten frei zu wählen, seien „strategische außenpolitische Interessen“ (ebd.). Damit wies der Sonderbeauftragte des Außenministeriums für Energiefragen nachdrücklich darauf hin, dass die USA zukünftig am europäischen Energiemarkt interessiert sein könnten, der bisher stark von Russland dominiert ist. Die Möglichkeit zukünftiger Exporte aus den USA sah der Energiepolitiker vor allem durch Überschüsse bei der Förderung von Erdgas. Zum einen ließe sich die US-Förderung noch deutlich ausbauen, zum anderen könnten die US-Unternehmen von den hohen Preisdifferenzen auf den verschiedenen Kontinenten profitieren. Die Bedingung dafür sei jedoch, dass Infrastrukturen ausgebaut würden, um das in Nordamerika geförderte Erdgas zu verflüssigen (Liquified natural gas - LNG) und zu verschiffen.

In den folgenden Monaten verließ die Debatte um die zunehmende Bedeutung der US-Energieförderung endgültig die energiepolitische Fachöffentlichkeit und schlug sich in zahlreichen Beiträgen einschlägiger Institutionen nieder. Exemplarisch seien hier nur die Position des Council on Foreign Relations (CFR) genannt. Anfang des Jahres 2014 veröffentlichten Robert Blackwill und Meghan O'Sullivan in Foreign Affairs, der Zeitschrift des CFR, den Beitrag America's Energy Edge, der vermutlich um den Jahreswechsel 2013/14 verfasst wurde. Beide 
Autoren arbeiteten als SicherheitsberaterInnen unter Präsident George Bush im National Security Council, wo sie für die Irak- und Afghanistan-Politik der Regierung zuständig waren. Beide sind überdies Senior Fellows des CFR. Meghan O'Sullivan lehrt als Professorin für Internationale Politik in Harvard, und im Februar 2015 nahm der Präsidentschaftskandidat der Republikaner Jeb Bush sie in sein außenpolitisches Beratungsteam auf.

Mit Blick auf die Öl- und Gasförderung in den USA gehen Blackwill und O'Sullivan (2014) davon aus, dass das Land auf dem Weg ist, sich in eine „Energiesupermacht“"zu verwandeln. Die förderbaren Energien werden ihnen zufolge die US-Wirtschaft ankurbeln und Washington neu gewonnenen Einfluss auf der ganzen Welt ermöglichen: „Der Boom der Öl- und Gasproduktion in Nordamerika, wird - verbunden mit anderen langfristigen Quellen der militärischen, wirtschaftlichen und kulturellen Stärke - den USA in den kommenden Jahren zu einer verstärkten globalen Führungsrolle verhelfen." (Ebd.) Wenn sich die bisherige Tendenz fortsetze, würde die US-Förderung „die globalen Öl- und Gaspreise unter Druck setzen“ (ebd.), prognostizieren die AutorInnen mehrere Monate vor dem globalen Preissturz. Die vielleicht „dramatischste geopolitische Folge“ (ebd.) der neuen Technologien sei, dass die nordamerikanische Produktion den globalen Preis für Erdöl senken könnte.

Ein dauerhaft niedriger Ölpreis würde, so Blackwill und O'Sullivan, grundsätzlich alle Staaten belasten, die Einnahmen aus Erdölexporten beziehen, während die Verbraucherländer von einer solchen Entwicklung profitierten. „Länder, die ihre Energieressourcen für außenpolitische Zwecke nutzen - in der Regel in einer Weise, die den Interessen der USA zuwiderläuft - werden ihren Einfluss schrumpfen sehen." (Blackwill/O’Sullivan 2014) Den größten Vorteil aus dieser Situation würden die USA ziehen, während „Moskau am meisten zu verlieren hat" (ebd.). Ein nachhaltiger Absturz des Ölpreises würde Russlands politisches System destabilisieren und Präsident Wladimir Putin könnte Einfluss verlieren, was neue Möglichkeiten für die Opposition eröffnen würde. An diesem Punkt kommen die AutorInnen auf mögliche Exporte von Flüssiggas (LNG) aus den USA zu sprechen. Nordamerikanisches Gas könne Russland als größten Einzelanbieter in Europa zwar nicht vollständig ersetzen. Europa werde aber stark profitieren, wenn es einen integrierten Gasmarkt schaffen und mehr LNG-Terminals bauen würde, um Flüssiggas aus den USA zu importieren. Zu diesem Zeitpunkt lagen in den USA bereits 20 Anträge auf Flüssiggasexport bei der US-Regierung vor.

Eine ähnliche Perspektive sehen Robert Blackwill und Meghan O'Sullivan für asiatische Länder. Die US-Verbündeten in Ostasien wie Japan, die Philippinen und Südkorea hätten die Möglichkeit, ihre Energieimporte aus den Vereinigten Staaten und Kanada zu erhöhen. Dass sie Erdöl und LNG über kürzere, direkte Seewege aus Nordamerika beziehen können, sollte ,auch für diese Länder“ - hier 
vergleichen die Autoren vermutlich die Situation mit Europa - einen wirtschaftspolitischen Vorteil darstellen.

Einen besonderen Einfluss sehen Blackwill und O'Sullivan hierbei auf den Verlauf der geplanten Freihandelsabkommen mit Asien und Europa, also TTIP (Transatlantic Trade and Investment Partnership) mit 28 EU-Staaten und TPP (Trans-Pacific Partnership) mit elf Ländern im asiatisch-pazifischen Raum und Lateinamerika. Sie verweisen auf die besondere US-Gesetzgebung zu Energieexporten, nach der eine Verschiffung von LNG in Länder mit Freihandelsabkommen sofort möglich ist. Für viele Länder in Europa und Asien sei dies ein starker Anreiz, sich auf die Freihandelsabkommen einzulassen. Insbesondere für Japan sei dies das entscheidende Motiv gewesen, sich an den TPP-Verhandlungen zu beteiligen.

Darüber hinaus heben sie hervor, dass der Technologievorsprung bei der unkonventionellen Förderung von Erdöl und Erdgas sich bereits in diplomatischen Initiativen niedergeschlagen habe. Sie empfehlen, die Regierung sollte diese Bemühungen noch ausweiten und sie in ihre „breitere Strategie mit den Verbündeten“ einbetten. Länder wie Polen und die Ukraine könnten darin unterstützt werden, ihre heimischen Schieferreserven intensiver zu nutzen. Hier folgt ein weiterer Hinweis auf Russland: „Weniger freundliche Anbieter“, etwa aus Russland, könnten mit den neuen Energieangeboten ausgebremst werden.

\section{Globale Neuordnung der Energielandkarte}

Aus dieser Perspektive betrachtet ergeben die Eckpunkte der "Weltumordnung“ (Candeias) zumindest aus der Perspektive der US-Außenpolitik ein durchaus sinnvolles Bild. Der Rohölpreis befindet sich in einem dauerhaften Tief. Den volkswirtschaftlichen Effekt der niedrigen Rohölpreise beschreibt der IWF als ein "gigantisches Konjunkturprogramm für die entwickelten Volkswirtschaften“ (IMF 2015), von dem vor allem die USA profitieren. Bereits in den vergangenen Jahren ermöglichten die extrem niedrigen Gaspreise auf dem US-Binnenmarkt eine deutlich erhöhte Konsumnachfrage der Privathaushalte durch Einsparungen von Energiekosten und Kostenvorteile für Unternehmen durch niedrigere NettoProduktionskosten. Die Kombination aus Niedrigzinspolitik, historisch niedrigen Inflationsraten sowie Steigerung der Produktivität durch Hochtechnologiepolitik führte zu wirtschaftlichem Wachstum und enormen Exportüberschüssen. Die aktuelle Außenpolitik zielt vor allem auf handelspolitische Maßnahmen, um künftig weltweit Absatzmärkte für eine hochproduktive US-Wirtschaft durch Freihandelsabkommen abzusichern.

Die aktuellen Verhandlungen orientieren auf ein globales T-Profil: Richtung Osten nach Asien, Richtung Westen zur Europäischen Union und Richtung 
Süden nach Lateinamerika. Die transatlantischen und transpazifischen Freihandelsabkommen (TTIP/TPP) knüpfen an das klassische Konzept des britischen Empire an, bei dem ein starker Staat weltweit Absatzmärkte erschließt und globale Handelsrouten sichert. Anders als beim „neuen Imperialismus“ der Bush-Ära ist sein Ziel nicht mehr die direkte Verwaltung im Sinne einzelner Energieunternehmen, sondern die Absicherung einer neuen wirtschaftlichen Konjunktur mit den USA im Zentrum: America's T-Strategy.

Wie schon im britischen Empire steht der hochtechnisierten Modernisierung der Binnenwirtschaft zwangsläufig ein „Kriegskapitalismus nach außen“ (Beckert 2014) gegenüber, wie sich exemplarisch am Umgang mit Russland, der Ukraine und der EU zeigen lässt. Als der Ukraine-Konflikt im Herbst 2013 begann, hatte die Ukraine drei Verträge mit internationalen Energieunternehmen angebahnt, die das Land mittelfristig von Erdgasimporten hätten unabhängig machen können (Tomlyak 2013). Alle Verträge kamen im Rahmen der nach dem Amtsantritt von Barack Obama eingerichteten Global-Shale-Gas-Initiative zustande, die von David Goldwyn, Koordinator für internationale Energie-Angelegenheiten, geleitet wurde. Die ukrainischen Projekte sollten in drei unterschiedlichen Regionen umgesetzt werden, im Schwarzen Meer vor der Küste der Krim, in der Ostukraine in der Region um Donezk und in der Westukraine, in der Gegend von Lwow. Insgesamt hatten die drei Unternehmen Investitionen in der Höhe von 32 Milliarden USDollar zugesagt, was auf die erwarteten Gewinne schließen lässt. Zusammen mit der bereits laufenden Gasförderung hätten die Projekte den gesamten Energiebedarf der Ukraine von 50 Milliarden Kubikmeter Erdgas pro Jahr annähernd decken können. Beteiligt waren die US-Unternehmen Exxon, Chevron und die britischniederländische Shell. Durch die Abtrennung der Krim und den Bürgerkrieg in der Ostukraine scheiterten alle drei Projekte (ausführlich Daniljuk 2014).

Als unmittelbare Reaktion auf die Ukraine-Krise planen die EU-Staaten, die russischen Energieimporte im Wert von etwa 400 Milliarden US-Dollar pro Jahr zumindest teilweise zu ersetzen. Bereits Anfang April 2014 verabschiedete der EU-USA-Energierat eine Erklärung, in der angekündigt wurde, „eine weitere Diversifizierung der Bezugsquellen und Lieferwege ... und eine höhere Produktion aus einheimischen Energieressourcen“ anzugehen. ${ }^{4}$ Dafür gründete der Europäische Rat auf seiner Sitzung im März 2015 die European Energy Union, in deren Rahmen alle 28 EU-Staaten eine gemeinsame Energiepolitik entwickeln sollen. Im Vorfeld fanden zahlreiche Sitzungen unter anderem mit US-Außenminister John Kerry und Vize-Präsident Joe Biden statt. Der Fokus liegt auf Importen von LNG und der Schaffung dafür geeigneter Strukturen. Dieses Projekt vergleichen

4 http://europa.eu/rapid/press-release_IP-14-365_de.doc, Zugriff: 4.11.2015. 
die Beteiligten mit der Tragweite der Europäischen Gemeinschaft für Kohle und Stahl (Montanunion) für die Formierung der Westeuropäischen Union in den 1950er Jahren (ausführlich Daniljuk 2015).

Im Zuge der Verhandlungen über das Freihandelsabkommen mit Kanada, CETA, senkte die EU-Kommission bereits deutlich die Kriterien für Schwerölimporte, wie sie in der europäischen „Kraftstoffqualitätsrichtlinie“ festgelegt werden sollten (Mahnke/Reimer 2014). Im Berliner Bundeskanzleramt fiel die Entscheidung, russische Gasimporte mittelfristig durch Lieferungen aus Nordamerika zu ersetzen, offensichtlich sehr schnell. Aus einem EU-Positionspapier zu CETA und TTIP, das fünf Tage vor dem oben erwähnten Treffen des EU-USA-Energierates veröffentlicht wurde (28.3.2014), geht hervor, dass Gas- und Ölimporte aus Kanada und den USA zukünftig zum wichtigsten Thema bei den Freihandelsgesprächen werden sollen. „TTIP wird dazu beitragen, die Sicherheit der Energieversorgung in der EU zu stärken“, heißt es darin mit Blick auf die Ukraine-Krise: „Eine solche Anstrengung beginnt man mit seinen engsten Verbündeten ". 2 Zudem sickerte aus Brüssel ein Entwurf für die TTIP-Verhandlungen vom September 2013 durch, mit dem der „freie Export von Rohöl- und Gasressourcen garantiert“ werden soll, darunter ausdrücklich LNG und die dazu gehörigen Infrastrukturen. ${ }^{6}$

Dem folgten eine Reihe von diplomatischen Initiativen zur Formierung einer European Energy Union und Konsultationen zwischen den USA und der EU, etwa am Rande der 51. Münchner Sicherheitskonferenz (MSC51). Auf dem Programm standen Gespräche über Importe von Flüssiggas und Erdöl aus der US-Fracking-Förderung. Unmittelbar vor der MSC15 startete die achte Verhandlungsrunde über TTIP in Brüssel. Auf der Tagungsordnung standen unter dem Begriff „Energiesicherheit für Europa“ ebenfalls die Verhandlungen über Fracking-Gas und Teersandöl aus Nordamerika. Die EU-Außenbeauftragte, die ehemalige italienische Außenministerin Federica Mogherini, nahm persönlich an beiden Treffen teil. Bereits vier Wochen zuvor, Anfang Januar 2015, hatte der EU-USA-Energierat unter Anwesenheit von US-Außenminister John Kerry in Brüssel getagt. ${ }^{7}$ Auf der Tagesordnung waren auch dort die energiepolitischen Aspekte des geplanten Freihandelsabkommens TTIP gestanden. Schließlich besuchte Bundeskanzlerin Angela Merkel im Mai 2015 die USA. Der Energiesektor, so die deutsche Bundeskanzlerin vor der US-Handelskammer, werde „möglicherweise die größten Gewinne“ aus TTIP ziehen. Das Projekt biete die Möglichkeit einer „vertieften Energiekooperation“ und einen Weg, Europa

5 Plusminus-Sendung des WDR vom 3.9.2014: CETA: Handel mit schädlichem Öl.

6 DG Trade: TTIP - non papers on raw materials and energy, Brussels, 20.9.2013.

7 http://www.euractiv.de/sections/energie-und-umwelt/ttip-mogherini-fordert-von-usaenergiekapitel-310608, Zugriff: 23.10.2015. 
von einer „unilateralen“ Versorgungsquelle unabhängig zu machen, erläuterte Merkel. Immerhin, so die Bundeskanzlerin, beziehe die EU bisher die Hälfte ihres Gasverbrauchs aus Russland. „Für uns wird es sehr wichtig sein, die TTIPVerhandlungen sehr schnell zu einem Abschluss zu bringen." ${ }^{\text {"8 }}$

Zwei Wochen später meldete sich aus Brüssel der neue Energiekommissar Maroš Šefčovič im Wall Street Journal zu Wort (Steinhauser 2015). Ein vereinfachter Export von Flüssiggas und Erdöl aus den USA sei eines der wichtigsten TTIP-Ziele, so Europas höchster Energiepolitiker. Wie schon Angela Merkel appellierte auch Šefčovič an die US-Regierung, die Energieexporte zu vereinfachen. Am folgenden Tag brach er zu einer Rundreise durch alle 28 EU-Mitgliedsstaaten auf, um in mündlichen Gesprächen die Vorteile der neuen European Energy Union zu erläutern.

\section{Zonen der Unsicherheit}

Betrachtet man die wissenschaftlichen Beschreibungen der sich verändernden „neuen Weltordnung" aus energiepolitischer Perspektive, dann lässt sich festhalten, dass das westliche Herrschaftssystem mit seinem kapitalbasierten, transnationalen und netzwerkartigen Vorgehen von Leo Panitch und Sam Gindin (2012) als American Empire absolut zutreffend beschrieben ist.

Bestimmte Kapitalfraktionen organisieren wirtschaftlich und politisch erfolgreiche Projekte in globalem Maßstab, ohne dass ein nationalistisches und zentralistisches Policy-Modell notwendig wäre, auch wenn die US-Politik dafür fraglos ein gewisses geografisches Zentrum innerhalb des angelsächsischen Machtblocks bildet. Was David Harvey und andere unter dem Eindruck des Irak-Krieges als „neuen Imperialismus“ beschrieben haben, erweist sich vor diesem Hintergrund dagegen als ein - zumindest teilweise - gescheitertes Projekt in einer bestimmten historischen Phase, die sich in etwa auf den Zeitraum von 2000 bis 2011 begrenzen lässt.

Die strategische Bedeutung des Greater Middle East hat für die US-Außenpolitik explizit abgenommen. Das direkte militärische Engagement ist nicht nur an den regionalen Bedingungen gescheitert, sondern im Rahmen der parallel verfolgten Strategie zur Energiesouveränität durch die Fracking-Förderung und die Regionalisierung des Energiebezugs in der „westlichen Hemisphäre“ reduzieren sich die energiepolitischen Handlungsnotwendigkeiten auf eine Sicherung der Handelswege, etwa mithilfe des US-Flottenstützpunkts in Bahrain.

Die Verantwortung für die weitere Entwicklung der Region hat Barack Obama ausdrücklich den regionalen Alliierten überlassen. Auch dieser transparente

8 http://www.bna.com/ttip-forged-end-n17179890190/, Zugriff: 23.10.2015. 
und plurale Politikansatz ist kennzeichnend für das American Empire (Panitch/ Gindin 2012). Allerdings waren die Verbündeten - sowohl die EU als auch die Golfstaaten, die Türkei und Ägypten - offensichtlich nicht in der Lage, mit dieser neuen Verantwortung umzugehen. Zu diesem „Regulierungsdefizit“ - den Zonen der Unsicherheit - mag auch beitragen, dass die historisch umkämpften Ressourcen, also zusätzliche Öl- und Gasangebote aus Libyen, dem Sudan oder Syrien, aufgrund der gegenwärtigen Versorgungssituation auf dem Weltmarkt nicht gerade dringend benötigt werden.

Verbunden mit der aktuellen Phase von America's T-Strategy erleben wir seit dem Jahr 2011 eine insgesamt aggressivere Außenpolitik, die etwa die EU in eine neue West-Ost-Konfrontation zwingt, wobei sich grundsätzlich zwei Integrationsperspektiven gegenüberstehen: die einer intensiveren transatlantischen Integration in den angelsächsischen Machtblock, in das American Empire; oder eine Perspektive „kontinentaler Integration“ in Richtung Russland, China und anderer Länder, mit denen die EU-Staaten immerhin auf dem Landweg verbunden sind.

Der Versuch, Russland als bisher wichtigsten Anbieter für fossile Energie zu verdrängen, und die Eskalation der verschiedenen Bürgerkriege zeigen jedoch auch: Innenpolitische Konflikte in Förderregionen führen nicht zwangsläufig zu niedriger Förderung von Rohstoffen und zu sinkenden Exporten. Die Beispiele Irak und Nigeria verdeutlichen, dass die westlichen Öl-Unternehmen unter bestimmten Bedingungen in der Lage sind, die Förderinseln zu sichern. Insbesondere die Förderung im Irak steigt seit zehn Jahren kontinuierlich; aber auch Nigeria konnte seine Öl-Exporte trotz einer Bürgerkriegssituation zuletzt wieder steigern. Gleichzeitig zeigt sich in Ländern wie dem Iran, Libyen, dem Sudan oder Syrien, in denen China als größter ausländischer Investor aktiv ist, dass die dortigen Bürgerkriege und internationalen Interventionen massive Verluste bei chinesischen Direktinvestitionen zur Folge haben.

Insofern bestehen sehr deutliche Hinweise darauf, dass America's T-Strategy sich vor allem gegen diejenigen potenziellen Konkurrenten richtet, die in der Phase der Globalisierung und vor allem durch die extrem hohen Rohstoffpreise von 2004 bis 2014 zu wirtschaftlicher Stärke gefunden haben, nämlich Russland und China. Ihr Versuch, etwa im Rahmen von BRICS, neue wirtschaftliche Stärke in politische Gestaltungsmacht umzusetzen, führt einerseits zu einem relativen Decline des American Empire insofern, als überhaupt neue globale Akteure auftreten, die eine stärkere multipolare Orientierung erzwingen. Andererseits hat sich das American Empire in absoluten Zahlen, was wirtschaftliche Stärke, militärische Reichweite, kulturelle Attraktivität und politische Gestaltungsfähigkeit betrifft, erneut als äußerst integrationsfähig und krisenresistent erwiesen. 


\section{Literatur}

Beckert, Sven (2014): King Cotton. Eine Geschichte des globalen Kapitalismus, München.

Blackwill, Robert D./O'Sullivan, Meghan (2014): America's Energy Edge. The Geopolitical Consequences of the Shale Revolution. In: Foreign Affairs, March/April. URL: https://www.foreignaffairs.com/articles/united-states/2014-02-12/americas-energy-edge, Zugriff: 23.10.2015.

Brown, Stephen P.A. (2013): The Shale Gas and Tight Oil Boom: U.S. States' Economic Gains and Vulnerabilities, CFR Energy Brief, October.

Candeias, Mario (2014): Weltumordnung. Wie die Konturen des Neuen allmählich sichtbar werden. In: Luxemburg 3/14: 20-23.

Cheney, Dick et al. (2001): Reliable, Affordable, and Environmentally Sound. Energy for America's Future. Report of the National Energy Policy Development Group, Washington.

Dammer, Anton R. (2004): Americas Oil Shale. A Roadmap for Federal Decision Making, Department of Energy, Washington.

Daniljuk, Malte (2014): Fracking, Freedom, Freihandel. In: Luxemburg 3/14: 38-45.

- (2015): TTIP: Freifahrt für Fracking. In: Blätter für deutsche und internationale Politik, 60(8): 17-20.

EIA (2011): Review of Emerging Ressources. U.S. Shale Gas and Shale Oil Plays, July 2011

Goldwyn, David L.: Making an Energy Boom Work for the U.S., New York Times, 12. November 2012

Grunwald, Michael/Eilperin, Juliet (2005): Energy Bill Raises Fears About Pollution, Fraud. In: Washington Post, 30.7.2005.

Harvey, David (2005): Der neue Imperialismus, Hamburg.

IMF, International Monetary Found (2015): World Economic Outlook. Uneven Growth: Short-and Long-Term Factors, April.

Johnson, Harry R./Crawford, Peter M./Bunger, James W. (2004): Strategic Significance of America's Oil Shale Resource, Volume I: Assessment of Strategic Issues, March 2004 Final Report, Office of Deputy Assistant Secretary for Petroleum Reserves, Washington.

Mahnke, Eva/Reimer, Nick (2014): EU öfnet Türen für Teersand aus Kanada. URL: http://www. klimaretter.info/politik/hintergrund/17362-eu-oeffnet-tueren-fuer-teersand-aus-kanada, Zugriff: 23.10.2015.

Obama, Barack (2011): Blueprint for a Secure Energy Future, White House, 30.3.2011.

- (2014): Remarks by the President at the United States Military Academy Commencement Ceremony, U.S. Military Academy-West Point, West Point, New York.

- (2015): National Security Strategy, February 2015, White House, Washington.

-/Biden, Joe (2008): New Energy for America, 2008, Energy.Gov

O'Sullivan, Meghan L. (2011): Iraqi Politics and Implications for Oil and Energy, Geopolitics of Energy Project, Belfer Center for Science and International Affairs, Cambridge/MA.

Panitch, Leo/Gindin, Sam (2012): The Making Of Global Capitalism: The Political Economy Of American Empire, London.

Steinhauser, Gabriele (2015): EU Wants U.S. to Lift Ban on Oil Exports. In: The Wall Street Journal, 17.5.2015. URL: http://www.wsj.com/articles/eu-wants-u-s-to-lift-ban-on-oil-exports-1431885401, Zugriff: 23.10.2015.

Stephens, Bret (2014): America in Retreat. The New Isolationism and the Coming Global Disorder, New York.

Tomlyak, Kyryl (2013): Future of Unconventional Projects in Ukraine in Light of last Agreements between Naftogaz and Gazprom. URL: http://shalegas.in.ua/en/ua-ru-agreements-and-unconventional-gas/, Zugriff: 23.10.2015.

van der Hoeven, Maria (2015): Broader Landscape for the European Energy Union. Conference on the Energy Union, Speech of IEA Executive Director in Riga, Latvia, 6.2.2015. 